\title{
Novel emerging treatments for NMOSD
}

\author{
Krzysztof Selmaj $^{1,2}$, Igor Selmaj ${ }^{2}$ \\ ${ }^{1}$ University of Warmia and Mazury, Olsztyn, Poland \\ ${ }^{2}$ Centre of Neurology, Lodz, Poland
}

\begin{abstract}
Neuromyelitis optica spectrum disorders (NMOSD) are inflammatory demyelinating diseases of the central nervous system (CNS) that cause optic neuritis, transverse myelitis, and some other CNS syndromes.

Recently, diagnosis and understanding of these diseases has been markedly enhanced by the discovery that serum autoantibodies that target aquaporin-4 (AQP4) are strongly associated with the disease. This spectrum includes also a potential subset of patients with a phenotype of NMOSD who have anti-myelin oligodendrocyte glycoprotein (MOG) antibody. Although steroids and immunosuppressive drugs have been widely used for NMOSD treatment, until recently there was no approved therapy for these diseases. With improved understanding of the pathophysiology of NMOSD, numerous new therapeutic strategies have recently been evaluated. The results of these studies, involving monoclonal antibodies ( $\mathrm{mAbs}$ ) inhibiting terminal complement protein cleavage interfering with interleukin-6 receptor (L-6 R) signaling and depleting CD19-positive B cells, have been published in recent months. All of these new therapeutics have shown a high degree of efficacy in diminishing NMOSD activity and inhibiting disability progression. At the same time, all these $\mathrm{mAbs}$ have demonstrated favorable safety and tolerability profiles, with a limited rate of adverse events. The first of these new drugs, eculizumab, have been approved in USA and Europe for NMOSD treatment within the last couple of months and it is expected that the other novel, effective and safe treatments for NMOSD will be approved in the near future.
\end{abstract}

Key words: neuromyelitis optica spectrum disorders, monoclonal antibodies, demyelinating diseases

(Neurol Neurochir Pol 2019; 53 (5): 317-326)

\section{Introduction}

Neuromyelitis optica spectrum disorders (NMOSD) are autoimmune inflammatory disorders of the central nervous system (CNS) characterised by bilateral or rapidly sequential optic neuritis and/or transverse myelitis. Other suggestive presentations include episodes of brainstem symptoms, in particular area postrema clinical syndrome. The disease can result in severe muscle weakness and paralysis, loss of vision, sensory loss, bladder dysfunction, neuropathic pain, and in the most severe cases respiratory failure [1]. The prevalence of NMOSD in Caucasians is relatively low at $0.5-10$ per 100,000 , but severe and frequently rapid impairment observed in patients can lead to an unfavourable prognosis, including death.

The mechanism of NMOSD is associated with the presence in the serum of patients' IgG1 antibodies against aquaporin-4 $(\mathrm{AQP} 4 \mathrm{Ab})$, the major water channel present within the CNS specifically on astrocyte endfeets at the blood-brain-barrier (BBB) [2]. This antibody can be found in more than
$80 \%$ of patients. The discovery of AQP4 Ab has contributed significantly to our understanding of the pathology of NMOSD, also allowing for a much more precise diagnosis. AQP4 $\mathrm{Ab}$ appears to have a pathogenic role in the mechanism of NMOSD [3]. Recombinant AQP4 Ab after passive transfer in rats has induced NMOSD-specific immunopathology [4]. Serum AQP4 Ab titers have been shown to correlate with clinical attacks and with the extent of spinal cord lesions on MRI $[5,6]$. In addition, serum AQP4 Ab titers have been shown to drop after immunosuppressive treatment, and to remain low during remissions [7]. This antibody, produced by T-helper dependent $B$ cells from the peripheral immune compartment, crosses the blood-brain-barrier (BBB) and interacts with AQP4 on astrocyte endfeet leading to astrocyte oedema and dysfunction. Subsequent inflammation involves complement activation, increased BBB permeability, and a massive influx of neutrophils and eosinophils [8]. Thus, inflammatory lesions in NMOSD are clearly different from CNS inflammation in multiple sclerosis (MS), where $\mathrm{T}$ and $\mathrm{B}$ cells constitute the majority of invading

Address for correspondence: Krzysztof Selmaj, Department of Neurology, University of Warmia and Mazury, Warszawska 30 Str., 10-082 Olsztyn, Poland, e-mail:kselmaj@gmail.com 
cells. These findings, demonstrating significant mechanistic differences between MS and NMOSD, have allowed for a definitive separation of these two clinical entities.

More recently, another antigen was found to be associated with AQP4 seronegative NMOSD. Antibodies against myelin oligodendrocyte glycoprotein (MOG) were detected in 4-11\% of seronegative patients [9]. Unlike AQP4 Ab, anti-MOG Ab did not induce astrocytic pathology. It was found that anti-MOG $\mathrm{Ab}$ led to demyelination with limited immune cell infiltration [10]. Anti-MOG Ab almost never co-exists with AQP4 Ab and occurs much more frequently in children than in adult patients.

Despite the discovery of AQP4 Ab and anti-MOG Ab, there are still patients who meet the clinical diagnostic criteria of NMOSD, but in whose sera the two Abs cannot be detected. The prerequisite of a NMOSD diagnosis requires the presence of optic nerve and spinal cord symptoms [11]. The current 2015 International Consensus Diagnostic Criteria for NMOSD are stratified according to the presence of $\mathrm{AQP} 4 \mathrm{Ab}$. For seropositive patients, they require the presence of at least one of the core manifestations, whereas for seronegative patients two manifestations including optic neuritis, myelitis or area postrema syndrome are required (Tab. 1). Recently, preliminary results have suggested the role of antibodies against glial fibrillary acidic protein (GFAP) in seronegative NMOSD [12].

\section{Current treatment of NMOSD}

Until recently, there was no approved treatment for NMOSD, and patients were restricted to off-label therapies bringing uncertain benefits. Based on its clinical course, NMOSD therapy can be divided into relapse treatment and preventive treatment.

\section{Relapse treatment}

For relapse, patients are usually treated with pulsed steroid therapy. Methylprednisolone at a dose of $1 \mathrm{~g}$ is given intravenously (IV) for 3-5 days but therapy can be extended in a very severe relapse [13]. Relapses that respond poorly to methylprednisolone can be treated with plasma exchange (PLEX), usually 5-7 procedures every other day. PLEX can also be used as a first line therapy for NMOSD relapse [14]. Since the progression of disability in NMOSD is mainly driven by relapses, it is critically important that NMOSD relapse is treated as early as possible and with the most effective procedure.

Shortly after steroid pulsed therapy or PLEX, patients usually are started with immunosuppressive therapies. To protect patients from disease progression before the immunosuppressive drugs start to work, prednisone or prednisolone can be given orally for up to six months. Relapses in NMOSD dependent on anti-MOG Ab usually respond better to steroid treatment than in AQP4 dependent disease [15]. Limited data indicates that intravenous immunoglobulins (IvIg) might show a benefit in NMOSD relapse treatment. In one study, the effectiveness of IvIg in the treatment of acute relapses in NMOSD was assessed in a retrospective review of 10 patients unresponsive to treatment with steroids with or without PLEX [16]. Improvement was noted in five of 11 (45.5\%) relapses, and the remaining relapses had no further worsening. The study concluded that IvIg may have a role in treating acute NMOSD relapses.

\section{Immunosuppressive treatment}

Based on the autoimmune mechanism of NMOSD involving the production of auto-antibodies against AQP4 and MOG, several immunosuppressive agents have been tested in this disease over the past 30 years. Unfortunately, on most occasions the studies only involved a small number of patients and were designed as open label trials. None of these drugs have been formally approved for NMOSD treatment.

Traditionally, Azathioprine (AZT) was widely used as a first line NMOSD treatment. AZT was recommended as

Table 1. IPND 2015 diagnostic criteria for NMOSD

\author{
NMOSD with AQP4-Ab \\ At least one core clinical characteristic plus positive test for AQP4-IgG using best available detection method* \\ Exclusion of alternative diagnoses \\ NMOSD without AQP4-Ab \\ At least two core clinical characteristics occurring as a result of one or more clinical attacks and meeting all of the following requirements: \\ 1. At least one core clinical characteristic must be optic neuritis, acute myelitis with LETM, or area postrema syndrome. \\ 2. Dissemination in space (two or more different core clinical characteristics). \\ 3. Additional MRI requirements, as applicable. \\ 4. Negative tests for AQP4-IgG using best available detection method* or testing unavailable. \\ 5. Exclusion of alternative diagnoses.
}

Core clinical characteristics: Optic neuritis; acute myelitis; area postrema syndrome (hiccups, nausea and vomiting); acute brainstem syndrome; symptomatic narcolepsy or acute diencephalic clinical syndrome with NMOSD-typical diencephalic MRI lesions; symptomatic cerebral syndrome with NMOSD-typical brain lesions.

*AQP4-IgG serology: cell-based assay is strongly recommended 
a first line preventive treatment by the EFNS panel on guidelines on diagnosis and management of NMO [17]. Several small studies have tested the efficacy of AZT alone, or in combination with steroids [18]. Some of these studies showed an approximately $70 \%$ reduction in the NMOSD relapse rate and reduced disability after several years of treatment. In a large retrospective review of the medical records of $103 \mathrm{AQP}-4$ antibody-positive NMOSD patients, $89 \%(\mathrm{n}=92)$ had reduced their median annualised relapse rates from 1.5 (IQR 0.6-4.0) to 0 (IQR $0-0.27, \mathrm{p}<0.00005$ ) during treatment. Sixty-one per cent $(n=63)$ remained relapse-free at a median follow-up of 18 months. Neurological function improved or stabilised in $78 \%$. However, the discontinuation rate was $47 \%$, reflecting the poor tolerability of this drug [19].

The other problem with AZT treatment in NMOSD is the delayed mechanism of action of this drug. AZT on average requires 3-6 months to demonstrate its effect on the immune system. Mitoxantrone [20], cyclophosphamide [21] and some other immunosuppressive treatments including methotrexate have demonstrated beneficial effects in NMOSD only in case reports and only in a limited number of patients. In addition, safety profiles of these drugs reduce the enthusiasm for their use in NMOSD. Little more data is available regarding Mycophenolate Mofetil (MMF). In a prospective study including 67 NMOSD seropositive and seronegative patients, MMF reduced the annual relapse rate by $49 \%$ and stabilised EDSS in $80 \%$ of patients within a 24 month observation period. There was no difference between seropositive and seronegative patients [22]. Several retrospective studies have assessed the efficacy of MMF in AQP4- and MOG- seropositive and double negative patients. In one of these studies [23], the median post-MMF annualised relapse rate was significantly lower than the pre-MMF annualised relapse rate $(0.0$ vs $1.5 ; \mathrm{p}<0.001)$. EDSS scores also significantly decreased after MMF treatment $(3.0 \mathrm{vs}$ $2.5 ; \mathrm{p}=0.005)$. Thirty-five patients $(60 \%)$ were relapse-free with a median treatment duration of 20 months, and EDSS scores were stabilised or improved in 53 patients (91\%). In a similar way to AZT, MMF requires an extended period of time to demonstrate its effect on the immune system, and a substantial number of patients discontinued treatment due to side effects.

\section{Intravenous immunoglobulins}

In recent years, intravenous immunoglobulins (IvIg), which have been proven to be effective in some other antibody-mediated autoimmune conditions including inflammatory demyelinating polyneuropathies [24], have attracted significant attention in terms of NMOSD treatment. IvIg have been shown to induce diminished activity on membrane-damaging components of the complement system, B cell activating factor (BAFF), and several other immune mechanisms including interference with antigen recognition, downregulation of cytokine secretion, adhesion molecules expression, and suppression of T-cell activation relevant to NMOSD [25]. Although popular views and practical experience are strong advocates for the use of IvIg in NMOSD, there is relatively little evidence to support these conclusions.

The first study to look into the role of IvIg treatment in NMOSD included eight patients [26]. Five experienced relapsing optic neuritis with or without myelitis, and the remaining three had relapsing longitudinal extensive transverse myelitis (LETM). After a total of 83 infusions (4-21 per patient) and a mean follow-up duration of 19.3 months (6-39 months), it was observed that the mean relapse rate had decreased from 1.8 in the 12 months pre-IvIg therapy to 0.006 during follow-up $(\mathrm{p}=0.0001)$, while the mean EDSS score had declined from $3.3 \pm 1.3$ to $2.6 \pm 1.5(\mathrm{p}=0.04)$. In another study [27], the use of IvIg was evaluated in preventing relapses in patients with NMOSD. Six NMOSD patients who were treated with an IvIg induction dose followed by infusions every 2-3 months were retrospectively analysed. ARR and EDSS pre- and post-IvIg were recorded. The median number of relapses and the median ARR were significantly reduced ( 8.0 to 1.0 and 0.75 to $0.15 ; \mathrm{p}<$ $0.05)$ during IvIg treatment. EDSS remained the same during four years of treatment.

\section{Cell depletion therapy}

The discovery of a pathological role of autoantibodies against AQP4 and MOG in NMOSD has prompted the application of $B$ cell depletion therapy which might help to eliminate antibody-producing cells and improve treatment of this disease. Rituximab (RTX), a chimeric monoclonal antibody against CD20, a pan-B cell surface marker, has been tested in NMOSD in several small or medium-sized open labelled studies [28, 29]. RTX very efficiently depleted B cells over a duration of 6-9 months. Importantly, the cells from early stages of B cell lineage development and plasma cells were preserved, securing immune homeostasis during RTX treatment.

Most of the RTX studies have shown its profound beneficial effect on ARR and on stabilisation and reduction of disability measured with EDSS. Some of these studies have even shown complete suppression of relapses over a 2-3 year period. A recently published meta-analysis analysed 26 studies, in which differences in the ARR ratio and EDSS score before and after RTX therapy were used as the main efficacy measures [30]. This meta-analysis involved 577 patients. Antibodies against aquaporin- 4 were present in 435 (75.39\%) patients. The findings suggested diminished mean ARR ratio after rituximab therapy by 1.56 ( $95 \% \mathrm{CI},-1.82$ to -1.29$)$. No significant correlation was detected between the outcome of ARR ratio change and the following variables: age at onset, duration of disease, follow-up time, dose of infusion or AQP4-IgG serostatus. The findings of this meta-analysis disclosed also a reduction in the mean EDSS score by -1.16 (95\% CI, -1.36 to -0.96) during RTX treatment. A total of 330 out of 528 patients $(62.9 \%)$ achieved relapse-free status. RTX showed acceptable tolerance, and there were no serious safety issues in NMOSD patients treated with RTX. All of these findings have led to increased off label use of RTX in NMOSD in recent years. 


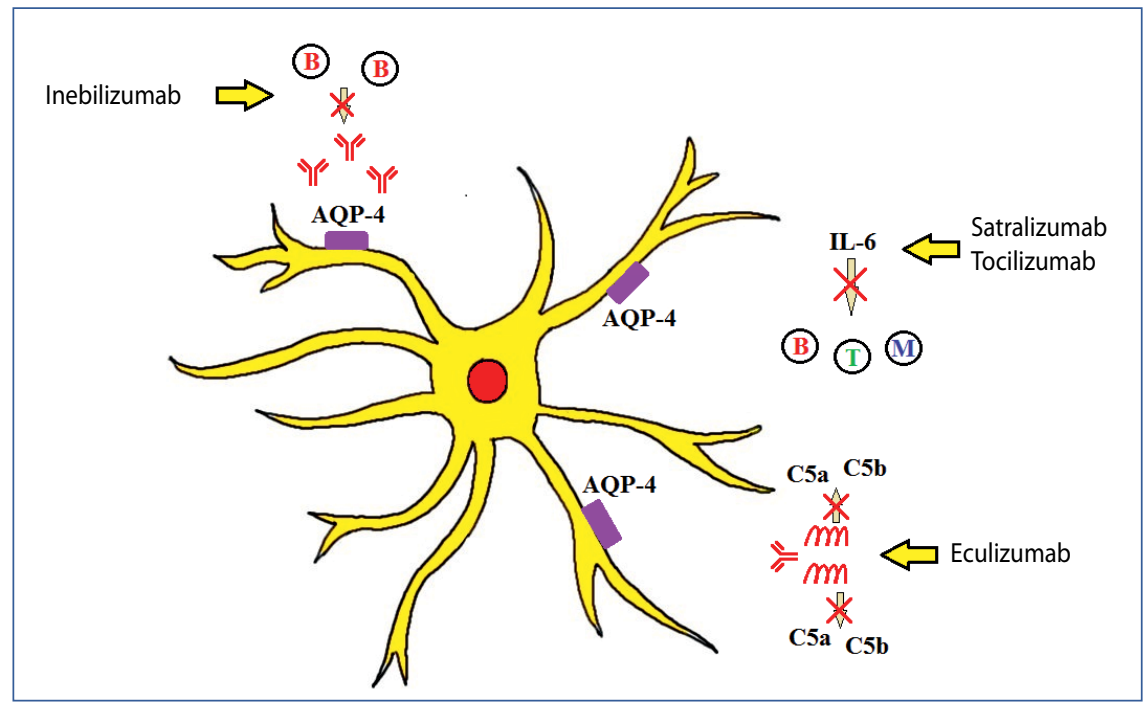

Figure 1. Emerging treatments of NMOSD

\section{Novel emerging treatments of NMOSD}

Progress in the understanding of NMOSD pathologic mechanisms has led to unprecedented attempts to intensify research into the development of new therapies in this disease in recent years. New therapeutic strategies have emerged which involve targeting novel molecules believed to be operating in the pathomechanism of NMOSD. These new strategies include the prevention of complement activation, interfering with IL6 receptor (IL6-R) signalling, and the depletion of AQP-4 and MOG antibodies producing cells (Fig. 1). More importantly, these new strategies were tested in studies designed as double blinded, randomised and controlled phase 2 and 3 trials. Within the last few months, very encouraging results of these studies have become available and have generated renewed hope of combatting this devastating disease. It is expected that the results of these recent studies will allow for the formal approval of all of these therapies for NMOSD in the near future.

\section{Eculizumab}

Eculizumab is a humanised monoclonal antibody which inhibits the terminal complement protein $\mathrm{C} 5$ and prevents its cleavage into $\mathrm{C} 5 \mathrm{a}$ and $\mathrm{C} 5 \mathrm{~b}$ fragments [31]. Preclinical data indicates that AQP4 Ab triggers the complement cascade [32], which leads to inflammation and the formation of a membrane attack complex. The membrane attack complex is implicated in astrocyte destruction and neuronal injury. C5a expresses proinflammatory activity, and $\mathrm{C} 5 \mathrm{~b}$ induces the formation of a membrane attack complex. Experimental studies have shown that complement inhibitor efficiently suppresses NMOSD development [33]. Eculizumab has been approved in the USA and Europe for paroxysmal nocturnal haemoglobinuria, atypical haemolytic uraemic syndrome, and myasthenia gravis.
In recently published results of a phase 3 , randomised, double-blind, placebo-controlled, time-to-event trial (PREVENT) it was shown that eculizumab significantly suppressed disease activity in 143 AQP4-Ab-positive patients with a moderate to severe course of the disease [34]. Patients were randomised 2:1 to either treatment with eculizumab or a placebo. The drug was administered intravenously at a dose of $900 \mathrm{mg}$ weekly for the first four doses; subsequently patients received a maintenance regimen of $1,200 \mathrm{mg}$ every two weeks until relapse or until the end of the trial. Immunosuppressive treatment used prior to study entry was allowed (with the exception of rituximab) and the group of immunosuppressive concomitant treatment was 108 patients out of the total group of 143. The primary efficacy endpoint was the first adjudicated relapse. The primary endpoint occurred much less often in the eculizumab group (3 of 96 patients - 3\%) than in the placebo group (20 of $47-43 \%$ ) (HR, 0.06; 95\% CI, 0.02 to $0.20 ; \mathrm{p}<0.001)$. At 48 weeks, $97.9 \%$ of patients receiving eculizumab were relapse-free, compared to $63.2 \%$ of patients receiving the placebo. Symptoms of most of the relapses were related to myelitis. Eculizumab was associated with a lower adjudicated annualised relapse rate after adjustment than the placebo, which represented the first secondary end-point, 0.02 and 0.35 , respectively $(\mathrm{p}<0.001)$. No conclusions could be made regarding the remaining secondary endpoints because the difference between groups for the next endpoint in the hierarchy, which was a change in the EDSS score, was not significant. The lack of between-group differences in disability progression resulted from the trial design precluding follow-up beyond six weeks after a single relapse. This implied that there were no benefits of the drug on disability progression during the short period of the trial. Patients from the eculizumab group had higher rates of upper respiratory tract infection and headache than did patients in 
the placebo group: 31 versus 19 events of upper respiratory tract infection per 100 patient-years, and 55 versus 38 headache events per 100 patient-years. One patient in the eculizumab group who was receiving concomitant azathioprine died from pulmonary empyema. Eculizumab increases the risk of meningococcal and encapsulated bacterial infection [35], and all patients received meningococcal vaccination prior to study entry. No cases of meningococcal infection were reported during the trial.

Eculizumab (Soliris) was approved by FDA for NMOSD treatment following an expedited six-month priority review in June 2019. EMA approved Eculizumab for treatment of $\mathrm{AQP} 4-\mathrm{Ab}$-positive patients with relapsing course of the disease in August 2019.

\section{Tocilizumab}

Tocilizumab was the first anti-IL6-R mAb tested in the treatment of NMOSD. The rationale for using tocilizumab in NMO was related to the potential role of IL- 6 in the mechanism of NMOSD [36]. This drug has been already used for the treatment of severe rheumatic arthritis patients.

Tocilizumab has been tested in NMOSD in several small open label studies and case reports $[37,38]$. These small series have demonstrated a beneficial effect in NMOSD. In one study [39], eight female patients with highly active AQP4-Ab-seropositive NMOSD $(n=6)$ and NMOSD patients $(n=2)$ whose disease had been resistant to previous medications including B-cell depletion, were switched to tocilizumab $(6-8 \mathrm{mg} /$ $\mathrm{kg}$ of body weight per dose). The patients were followed up for 30.9 months after switching to tocilizumab. Two of the eight patients received add-on therapy consisting of monthly corticosteroid pulses or azathioprine. During tocilizumab treatment, the median annualised relapse rate significantly decreased from 4.0 in the year before tocilizumab therapy to $0.4(\mathrm{p}=0.008)$, and the median EDSS score significantly decreased, from 7.3 to $5.5(\mathrm{p}=0.03)$. Active magnetic resonance imaging lesions were seen in $6 / 8$ patients at tocilizumab initiation and in $1 / 8$ patients at the last magnetic resonance imaging. Three patients remained relapse-free during tocilizumab treatment. The AQP4-Ab titers $(\mathrm{p}=0.02)$ and pain levels $(p=0.02)$ dropped significantly during tocilizumab treatment. Adverse effects included moderate cholesterol elevation in $6 / 8$ patients, infections in $4 / 8$, deep venous thrombosis in $1 / 8$, and neutropenia in $1 / 8$.

Tocilizumab development for NMOSD treatment has been delayed since another anti-IL6-R mAb, satralizumab, was designed to improve pharmacokinetics of IL6-R binding and provide better efficacy (see below). However, at the recent ECTRIMS meeting in Stockholm, the results were presented of an investigator-initiated study (Tango) in China [40]. This was a randomised, open-label, parallel-group study comparing tocilizumab with azathioprine. Patients $(n=118)$ were randomly assigned $1: 1$ to receive $8 \mathrm{mg} / \mathrm{kg}$ intravenous tocilizumab monthly or $2-3 \mathrm{mg} / \mathrm{kg}$ oral azathioprine daily. Treatment was administered in conjunction with a gradual discontinuation of the previous treatments, followed by monotherapy for 12 months; $85 \%$ of patients were seropositive for AQP-4. The primary endpoint was the time to first relapse. After a mean observation period of 48 weeks, the percentages of relapse-free patients were $91.5 \%$ in the tocilizumab group and $67.8 \%$ in the azathioprine group ( $\mathrm{HR}=0.32,95 \% \mathrm{CI}$ $0.14-0.70, p=0.004)$. Sustained reduction in disability was more likely among patients treated with tocilizumab than in patients with azathioprine $(\mathrm{HR}=0.34,95 \% \mathrm{CI} 0.13-0.90$, $\mathrm{p}=0.03)$. Serum levels of anti-AQP4-ab were reduced significantly, by $42 \%$ with tocilizumab compared to $15 \%$ with azathioprine $(p=0.03)$. In the azathioprine group, there was a higher frequency of lymphopenia ( $46 \%$ vs $7 \%$ ) and anaemia ( $27 \%$ vs $41 \%$ ) than in the tocilizumab group. During the Tango study, two patients died (one in each arm): in tocilizumab because of a severe recurrence of NMOSD, and in azathioprine because of meningoencephalitis. In both groups, there was an increased number of patients with elevated transaminases $(31 \%$ and $46 \%$ ) respectively for tocilizumab and azathioprine.

\section{Satralizumab}

Satralizumab is a humanised IgG2 subtype recombinant anti-IL6-R monoclonal antibody [41]. IL-6 is a pro-inflammatory pleiotropic cytokine produced by a large number of cell types, including $\mathrm{T}$ and $\mathrm{B}$ lymphocytes, monocytes and fibroblasts [42]. IL-6 plays a role in several immunopathological processes such as T-cell activation, induction of immunoglobulin secretion, and enhancing macrophage activity, and it has been implicated in the mechanism of NMOSD [43]. IL-6 specifically contributes to the development of autoimmunity by promoting the generation of Th17 cell lineage [44]. Th17 cells are recognised as the primary $\mathrm{T}$ cell sub-population contributing to the development of autoimmune conditions. IL- 6 has been found to be significantly elevated in the serum and cerebrospinal fluid of patients with NMOSD; it induces $\mathrm{AQP} 4-\mathrm{Ab}$ production by plasmablasts, and thus represents a novel therapeutic target for NMOSD.

The molecule of satralizumab was designed to improve pharmacokinetics of anti-IL6-R Ab by applying so-called 'antibody recycling technology' [45]. This technology leads to increased dissociation of anti-IL6-R Ab from IL6-R within the acidic environment of the endosome, while maintaining its binding affinity to IL6-R in plasma. Thus, in the endosome acidic environment, IL6- $R$ Ab after degradation of IL6- $R$ is dissociated from this complex and can again bind another IL6- $\mathrm{R}$ in the plasma, increasing its efficacy of IL6- $\mathrm{R}$ elimination.

Satralizumab efficacy in NMOSD was evaluated in 83 patients in a randomised, double-blind, phase 3 study (SAkuraSky) [46]. AQP4-positive patients represented $66.3 \%$ and AQP4-negative patients $33.7 \%$. In this study, satralizumab was compared to a placebo as an add-on to baseline treatment with a stable dose of immunosuppressive and/or corticosteroids. 
Subjects were randomised to satralizumab (120 mg s.c.) or placebo administered at weeks $0,2,4$, and Q4W thereafter. The primary endpoint was time to first protocol-defined relapse (PDR), adjudicated by a clinical endpoint committee. Pre-specified subgroup analyses included assessing the response to treatment by AQP4-Ab serostatus, baseline treatment, and region. Satralizumab showed a $79 \%$ risk reduction of PDR compared to placebo in the NMOSD AQP4-Ab positive subgroup (HR, 0.21; 95\% CI, 0.06--0.75). At weeks 48 and 96, the percentages of relapse-free patients were $91.5 \%$ (95\% CI, 69.6\%-97.8\%) and 91.5\% (95\% CI, 69.6\%-97.8\%) with satralizumab and $59.9 \%(95 \% \mathrm{CI}, 36.3 \%-77.3 \%)$ and $53.3 \%(95 \%$ CI, 29.3\%-72.4\%) with placebo, respectively. However for the NMOSD AQP4-Ab negative patients, satralizumab showed a risk reduction of PDR that was significantly lower compared to the AQP4 Ab positive group, 34\% compared to the placebo group (HR, 0.66; 95\% CI, 0.20-2.23), and the percentages of relapse-free patients at weeks 48 and 96 were $84.4 \%$ (95\% CI, $50.4 \%-95.9 \%$ ) and $56.3 \%$ (95\% CI, 24.2\%-79.2\%) with satralizumab, and $75.5 \%$ (95\% CI, 41.6\%-91.4\%) and 67.1\% (95\% CI, $34.2 \%-86.2 \%$ ) with placebo, respectively.

More recently, results have become available for satralizumab monotherapy in NMOSD. In the SAkuraStar study, the efficacy and safety of satralizumab was compared to placebo for relapse prevention in patients with NMOSD [47]. In this phase 3, double-blind, placebo-controlled study, 95 patients were randomised 2:1 to satralizumab (120 mg s.c.) or placebo, administered at weeks 0, 2, 4 and every four weeks thereafter. Unlike the SAkuraSky study, concomitant immunosuppressant medications were prohibited. Patients with AQP-4 antibodies represented $65.1 \%$ in the satralizumab group and $71.9 \%$ in the placebo group. All patients had had $\geq 1$ documented relapse, including first attack, in the year prior to screening. The primary endpoint was time to first protocol-defined relapse (PDR) adjudicated by a clinical endpoint committee. Satralizumab monotherapy significantly reduced the risk of PDR by $55 \%$ compared to placebo (HR 0.45; 95\% CI 0.23-0.89; $\mathrm{p}=0.018$ ). The percentages of relapse-free patients at week 48 were $76.1 \%$ in the satralizumab group, and $61.9 \%$ in the placebo group. At week 96, these values were $72.1 \%$ and $51.2 \%$, respectively. As in the SAkuraSky study, in the group of patients without AQP-4 Ab, satralizumab showed significantly less effect on the proportion of relapse-free compared to the AQP- $4 \mathrm{Ab}$ positive patients, and the difference between satralizumab and placebo was not significant. Satralizumab was well tolerated, and similar proportions of patients in the satralizumab and placebo groups experienced adverse events. Rates of serious infections were similar between groups. No deaths or anaphylactic reactions were observed with satralizumab or placebo treatment.

The clear difference in satralizumab efficacy between AQP4 Ab-positive patients and AQP4 Ab-negative patients observed in the SAkuraSky and SAkuraStar studies will require further analysis.

\section{Inebilizumab}

Inebilizumab is a humanised mAb of IgG1 subtype directed against the extracellular B cell marker CD19 leading to depletion of a broad range of $B$ cells, including autoantibody-secreting plasmablasts and CD19-expressing plasma cells [48]. Inebilizumab induces a cytotoxic T-lymphocyte response and a strong antibody dependent cell cytotoxicity (ADCC) against B cells.

Inebilizumab was evaluated in a phase 3 , double-blind, randomised, placebo-controlled trial (N-MOmentum) in 231 NMOSD patients, both AQP4 Ab-positive (91\%) and AQP4 Ab-negative (9\%) [49]. Enrollment of AQP4 Ab-negative patients required approval of an eligibility committee that confirmed the entry criteria. Participants were randomised 3:1 to either treatment with inebilizumab, $600 \mathrm{mg}$ iv in two doses of $300 \mathrm{mg}$ each two weeks apart, or a placebo, with no further doses occurring after day 15. Concurrent treatment with other immune suppressants was prohibited.

This means that inebilizumab was the first monotherapy tested in NMOSD free from the confounding influence of other background immunosuppressive treatments. B cells were depleted within approximately four weeks of treatment initiation, and this depletion was sustained throughout the randomised, controlled period of the study. The patients were followed for 28 weeks, after which time the blinded control period was stopped early for efficacy. The primary outcome measure was time to first adjudicated relapse. Following the blinded period, patients were given the option of entering an open-label extension period, in which they received $300 \mathrm{mg}$ of inebilizumab every six months. Inebilizumab met the primary efficacy endpoint with a $77 \%$ reduction in the risk of developing an NMOSD relapse when compared to placebo in AQP4-Ab seropositive patients after 28 weeks of treatment (HR: 0.227; $\mathrm{p}<0.0001$ )

A similar effect on relapse risk (73\% reduction) was observed in the total inebilizumab-treated patient population, inclusive of AQP4-Ab seronegative patients, (HR: 0.272; $\mathrm{p}<0.0001)$. At 28 weeks, at the end of the randomised-controlled period, $89 \%$ of AQP4-Ab seropositive patients treated with inebilizumab were relapse-free, versus $58 \%$ in the placebo group. Inebilizumab had also met most of the secondary endpoints. Reduction of disability worsening measured with EDSS in the inebilizumab-treated patients was significantly lower, $15.5 \%$, than in the placebo group where it was $33.9 \%$, $(p=0.0049)$. In inebilizumab-treated patients, the reduction in NMOSD-related hospitalisations was significantly lower, $5.7 \%$ of patients, versus placebo $14.3 \%(\mathrm{p}=0.01)$. In this study, MRI was also used as a secondary endpoint and showed a reduction in the frequency of cumulative total active MRI lesions in inebilizumab-treated patients (45.4\% patients) versus placebo (57.1\%) $(\mathrm{p}=0.0034)$. Visual acuity, another secondary endpoint, did not demonstrate a statistically significant difference 
between inebilizumab-treated patients and placebo. Inebilizumab demonstrated favourable safety and tolerability profiles, with an adverse event rate similar to that of the placebo. The rate of infusion-related reactions was low in both arms. The rates of serious and/or $\geq$ Grade 3 severity adverse events were similar in the inebilizumab (10.3\%) and placebo (14.3\%) groups. Two deaths occurred in the open-label period: one related to a severe NMOSD relapse, and the other related to a brain event of unclear aetiology without a definite diagnosis.

Based on data from the pivotal N-MOmentum study, the FDA has granted Breakthrough Therapy Designation (BTD) for the development of inebilizumab for the treatment of NMOSD.

Breakthrough Therapy Designation is designed to expedite the development and regulatory review of medicines intended to treat a serious condition that have shown encouraging early clinical results which may demonstrate substantial improvement on a clinically significant endpoint over available medicines.

\section{Future perspectives}

The pathogenic role of AQP4-Ab in NMOSD is associated with the production of AQP4-Ab by peripheral plasma cells, which can enter the CNS and bind to AQP4 on perivascular astrocytes. This binding initiates activation of the terminal complement complex and the induction of inflammatory lesion formation. Impaired blood-brain barrier function allows for a massive influx of neutrophils and eosinophils into the CNS of NMOSD patients. Neutrophil counts are elevated in CSF in about $60 \%$ of NMOSD patients during relapse, and about $20 \%$ during remission [50]. Eosinophils are also present in the CSF of NMOSD patients. Degranulation of these cells and the release of several toxic proteins and enzymes provide a direct mechanism of damage to astrocytes, followed by oligodendrocyte injury and neuronal death [51].

Future NMOSD therapies will need to address the inhibition of AQP4-Ab and prevention of neutrophils and eosinophils activation leading to CNS infiltration.

In vitro and in vivo studies have demonstrated that AQP4-Ab deglycosylation or cleavage reduce the complement-dependent cytotoxicity and the antibody-dependent cell-mediated cytotoxicity, leading to decreased astrocyte damage and reduced development of inflammation [52]. Similarly, IgG-degrading enzyme produced by Streptococcus pyogenes efficiently cleaved AQP4-Ab in mice in vivo and greatly reduced lesion formation in an experimental mice model of NMOSD [53]. The bacteria-derived endoglycosidases may target AQP4-Ab and reduce its pathogenicity by inhibition of the AQP4-Ab binding to AQP4 and preventing NMOSD pathology. Another strategy to inhibit interaction between AQP4-Ab with AQP-4 protein involved Aquaporumab which is a synthetic IgG that competes with AQP4-Ab for AQP 4 binding [54]. In contrast to pathogenic AQP4-Ab, the mutated $\mathrm{Fc} \gamma$ portion of Aquaporumab does not activate the antibody-dependent complement and cell-dependent mediated cytotoxicity. Its competitive inhibition of AQP4-Ab binding depends also on the greater affinity of aquaporumab to the AQP4 protein, compared to that of pathological AQP4-Ab. Its efficacy to compete with AQP4-Ab binding has already been proven in a preclinical study [55].

The dominant presence of neutrophils in inflammatory infiltrates of the CNS, and the proven role of these cells in NMOSD lesions formation, points at their inhibition as a new strategy of NMOSD treatment.

This concept was supported by findings that intracerebral injection of AQP-4 Ab in neutropenic mice induced less inflammation and demyelination than in mice with normal neutrophil counts [56]. The potential utility of neutrophil protease inhibitors might have particular significance in this regard [57]. It has already been shown that Sivelestat, an inhibitor of neutrophil elastase, demonstrated a beneficial effect in animal models of NMOSD, as evidenced by reduced NMOSD lesion formation [56]. Sivelestat was applied intraperitoneally or intracerebrally either alone or in combination with cathepsin $\mathrm{G}$ inhibitor. In addition to its inhibition of proteolytic activity, Sivelestat also reduced the production of inflammatory cytokines and suppressed neutrophil-induced capillary permeability and leukocyte kinetics in other conditions [58].

Eosinophil infiltration is another prominent feature of NMOSD lesions, and eosinophils have been found to be elevated in the CSF of NMOSD patients [59]. Accordingly, hypoeosinophilic mice showed diminished potential for NMOSD development. Eosinophil inhibition, either by anti-IL-5 or gene depletion, led to reduced lesion severity in experimental models of NMOSD.

These findings confirm the involvement of eosinophils in NMOSD's pathogenesis, and suggests the therapeutic utility of eosinophil-targeted drugs [60]. The inhibition of eosinophil degranulation has demonstrated promising results in animal models of NMOSD. The histamine $\mathrm{H} 1$ receptors antagonists have been shown to influence eosinophil activity. Cetirizine, a selective antagonist of the $\mathrm{H} 1$ receptor, was administered orally before and during AQP4-Ab intracerebral injection and significantly reduced eosinophil infiltrates and lesion formation in mice. These results prompted the testing of cetirizine in a pilot, open-label, add-on trial to standard therapy for 16 NMOSD patients. Cetirizine was administered at a dose of $10 \mathrm{mg}$ daily. After one year of treatment, ARR was reduced fourfold in these patients [61]. Thus, antihistaminic drugs affecting eosinophilic function might be beneficial as an add-on therapy in NMOSD treatment.

Another future strategy for NMOSD treatment might be associated with an attempt to enhance apoptotic death of plasma cells. This strategy might reduce the number of cells producing pathogenic $\mathrm{AQP} 4-\mathrm{Ab}$ and demonstrate benefit for NMOSD patients. 
Table 2. Results of primary findings for novel NMOSD treatment

\begin{tabular}{|c|c|c|c|c|}
\hline & Eculizumab & Inebilizumab & $\begin{array}{c}\text { Satralizumab } \\
\text { (SAkuraSky) } \\
\text { (SakuraStar) }\end{array}$ & Tocilizumab \\
\hline Mechanism of action & Anti-C5 & Anti-CD19 & $\begin{array}{c}\text { Anti-IL6-R } \\
\text { Ab recycling }\end{array}$ & Anti-IL6-R \\
\hline Patients, number & 143 & 230 & $\begin{array}{l}83 \\
95\end{array}$ & 118 \\
\hline \multicolumn{5}{|l|}{ Status AQP-4 Ab } \\
\hline - positive & 143 & 212 & $\begin{array}{l}55 \\
62\end{array}$ & 100 \\
\hline - negative & 0 & 18 & $\begin{array}{l}28 \\
33\end{array}$ & 18 \\
\hline Placebo & + & + & $\begin{array}{l}+ \\
+\end{array}$ & AZT \\
\hline \multicolumn{5}{|l|}{ Concomitant } \\
\hline Immunosuppression & + & - & + & $+/-$ \\
\hline \multirow[t]{2}{*}{ Relapse free } & $96.1 \%$ & $87.6 \%$ & $91.5 \%(\mathrm{AQP}+)$ & $91.5 \%$ \\
\hline & $(96 w)$ & $(28 w)$ & $\begin{array}{c}72.1 \%(\mathrm{AQP}+) \\
(96 \mathrm{w})\end{array}$ & $(48 w)$ \\
\hline Relapse reduction & $93.1 \%$ & $73 \%$ & $79 \%(A Q P+)$ & NA \\
\hline (HR) & $(0.06)$ & $(0.272)$ & $\begin{array}{c}74 \%(\mathrm{AQP}+) \\
(0.21)\end{array}$ & \\
\hline Disability risk reduction & NS & 0.371 & NA & 0.34 \\
\hline (OR) & & & NA & \\
\hline
\end{tabular}

$\mathrm{HR}$ - hazard ratio; $\mathrm{OR}$ - odds ratio; $\mathrm{W}$ - weeks; NS - non significant; NA — not available

Bortezomib is a selective inhibitor of the $26 \mathrm{~S}$ proteasome subunit leading to enhanced cell death. Bortezomib has been tested in an open-label study including five NMOSD AQP4-Ab-positive patients. All patients were refractory to previous therapies, and two of them were resistant to RTX. Four of these five patients remained stable or improved within 12 months of the study. Patients treated with bortezomib had a lower plasma cell count and diminished levels of serum AQP4-Ab [62].

\section{Conclusions}

Recent years have witnessed unprecedented progress in the understanding and treatment of NMOSD (Tab. 2). 2019 has been called the Year of NMOSD. The discovery of the pathogenic role of AQP4-Ab and anti-MOG Ab has allowed the targeting of the basic immune mechanism of this disease.

The exciting findings of the recent randomised and controlled trials have provided a realistic hope that the era of unproved therapies in NMOSD will come to the end.

The findings from studies with novel drugs targeting complement activation, interfering with IL6-R activation and depleting antibody-producing plasma cells, should soon bring about a new and effective treatment of this devastating disease. These results are very welcome for NMOSD patients who have been relegated to off-label therapies with uncertain benefits for many years. The approval of new therapies for NMOSD will undoubtedly prove to be another breakthrough in modern neurology.

\section{References}

1. Flanagan EP, Weinshenker BG. Neuromyelitis optica spectrum disorders. Curr Neurol Neurosci Rep. 2014; 14(9): 483, doi: 10.1007/ s11910-014-0483-3, indexed in Pubmed: 25027264.

2. Lennon VA, Wingerchuk DM, Kryzer TJ, et al. A serum autoantibody marker of neuromyelitis optica: distinction from multiple sclerosis. Lancet. 2004; 364(9451): 2106-2112, doi: 10.1016/S0140-6736(04)17551-X, indexed in Pubmed: 15589308.

3. Hinson SR, Pittock SJ, Lucchinetti CF, et al. Pathogenic potential of IgG binding to water channel extracellular domain in neuromyelitis optica. Neurology. 2007; 69(24): 2221-2231, doi: 10.1212/01. WNL.0000289761.64862.ce, indexed in Pubmed: 17928579.

4. Bennett JL, Lam C, Kalluri SR, et al. Intrathecal pathogenic anti-aquaporin-4 antibodies in early neuromyelitis optica. Ann Neurol. 2009; 66(5): 617-629, doi: 10.1002/ana.21802, indexed in Pubmed: 19938104.

5. Takahashi T, Fujihara K, Nakashima I, et al. Anti-aquaporin-4 antibody is involved in the pathogenesis of NMO: a study on antibody titre. Brain. 2007; 130(Pt 5): 1235-1243, doi: 10.1093/brain/awm062, indexed in Pubmed: 17449477.

6. Jarius $\mathrm{S}$, Aboul-Enein $\mathrm{F}$, Waters $\mathrm{P}$, et al. Antibody to aquaporin-4 in the long-term course of neuromyelitis optica. Brain. 2008; 131(Pt 
11): 3072-3080, doi: 10.1093/brain/awn240, indexed in Pubmed: 18945724.

7. Papadopoulos M, Verkman AS. Aquaporin 4 and neuromyelitis optica. The Lancet Neurology. 2012; 11(6): 535-544, doi: 10.1016/s14744422(12)70133-3.

8. Bradl M, Reindl M, Lassmann H. Mechanisms for lesion localization in neuromyelitis optica spectrum disorders. Curr Opin Neurol. 2018; 31(3): 325-333, doi: 10.1097/WC0.0000000000000551, indexed in Pubmed: 29465432.

9. Sato DK, Callegaro D, Lana-Peixoto MA, et al. Distinction between MOG antibody-positive and AQP4 antibody-positive NMO spectrum disorders. Neurology. 2014; 82(6): 474-481, doi: 10.1212/ WNL.0000000000000101, indexed in Pubmed: 24415568.

10. Ikeda K, Kiyota N, Kuroda $\mathrm{H}$, et al. Severe demyelination but no astrocytopathy in clinically definite neuromyelitis optica with anti-myelin-oligodendrocyte glycoprotein antibody. Mult Scler. 2015; 21(5): 656-659, doi: 10.1177/1352458514551455, indexed in Pubmed: 25257613.

11. Wingerchuk DM, Banwell B, Bennett JL, et al. International Panel for NMO Diagnosis. International consensus diagnostic criteria for neuromyelitis optica spectrum disorders. Neurology. 2015; 85(2): 177-189, doi: 10.1212/WNL.0000000000001729, indexed in Pubmed: 26092914.

12. Sechi E, Morris PP, McKeon A, et al. Glial fibrillary acidic protein IgG related myelitis: characterisation and comparison with aquaporin-4-lgG myelitis. J Neurol Neurosurg Psychiatry. 2019; 90(4): 488-490, doi: 10.1136/jnnp-2018-318004, indexed in Pubmed: 30032117.

13. Abboud H, Petrak A, Mealy M, et al. Treatment of acute relapses in neuromyelitis optica: Steroids alone versus steroids plus plasma exchange. Mult Scler. 2016; 22(2): 185-192, doi: 10.1177/1352458515581438, indexed in Pubmed: 25921047.

14. Watanabe S, Nakashima I, Misu T, et al. Therapeutic efficacy of plasma exchange in NMO-IgG-positive patients with neuromyelitis optica. Mult Scler. 2007; 13(1): 128-132, doi: 10.1177/1352458506071174, indexed in Pubmed: 17294622.

15. Reindl M, Waters P. Myelin oligodendrocyte glycoprotein antibodies in neurological disease. Nat Rev Neurol. 2019; 15(2): 89-102, doi: 10.1038/s41582-018-0112-x, indexed in Pubmed: 30559466.

16. Elsone L, Panicker J, Mutch K, et al. Role of intravenous immunoglobulin in the treatment of acute relapses of neuromyelitis optica: experience in 10 patients. Mult Scler. 2014; 20(4): 501-504, doi: 10.1177/1352458513495938, indexed in Pubmed: 23986097.

17. Sellner J, Boggild M, Clanet M, et al. EFNS guidelines on diagnosis and management of neuromyelitis optica. Eur J Neurol. 2010; 17(8): 1019-1032, doi: 10.1111/j.1468-1331.2010.03066.x, indexed in Pubmed: 20528913.

18. Kimbrough DJ, Fujihara K, Jacob A, et al. GJCF-CC\&BR. Treatment of Neuromyelitis Optica: Review and Recommendations. Mult Scler Relat Disord. 2012; 1(4): 180-187, doi: 10.1016/j.msard.2012.06.002, indexed in Pubmed: 24555176.

19. Elsone L, Kitley J, Luppe S, et al. Long-term efficacy, tolerability and retention rate of azathioprine in 103 aquaporin- 4 antibody-positive neuromyelitis optica spectrum disorder patients: a multicentre retrospective observational study from the UK. Mult Scler. 2014; 20(11): 1533-1540, doi: 10.1177/1352458514525870, indexed in Pubmed: 24647557.

20. Cabre P, Olindo S, Marignier R, et al. Aegis of French National Observatory of Multiple Sclerosis. Efficacy of mitoxantrone in neuromyelitis optica spectrum: clinical and neuroradiological study. J Neurol Neurosurg
Psychiatry. 2013; 84(5): 511-516, doi: 10.1136/jnnp-2012-303121, indexed in Pubmed: 23138769.

21. Awad A, Stüve 0. Cyclophosphamide in multiple sclerosis: scientific rationale, history and novel treatment paradigms. Ther Adv Neurol Disord. 2009; 2(6): 50-61, doi: 10.1177/1756285609344375, indexed in Pubmed: 21180630.

22. Montcuquet A, Collongues N, Papeix C, et al. NOMADMUS study group and the Observatoire Français de la Sclérose en Plaques (OFSEP). Effectiveness of mycophenolate mofetil as first-line therapy in AQP4-IgG, MOG-IgG, and seronegative neuromyelitis optica spectrum disorders. Mult Scler. 2017; 23(10): 1377-1384, doi: 10.1177/1352458516678474, indexed in Pubmed: 27885065.

23. Huh SY, Kim SH, Hyun JW, et al. Mycophenolate Mofetil in the Treatment of Neuromyelitis Optica Spectrum Disorder. JAMA Neurology. 2014; 71(11): 1372, doi: 10.1001/jamaneurol.2014.2057.

24. Hughes RAC, Donofrio P, Bril V, et al. ICE Study Group. Intravenous immune globulin ( $10 \%$ caprylate-chromatography purified) for the treatment of chronic inflammatory demyelinating polyradiculoneuropathy (ICE study): a randomised placebo-controlled trial. Lancet Neurol. 2008; 7(2): 136-144, doi: 10.1016/S1474-4422(07)70329-0, indexed in Pubmed: 18178525.

25. Dalakas M. IVIg in other autoimmune neurological disorders: current status and future prospects. J Neurol. 2008; 255 Suppl 3: 12-16, doi: 10.1007/s00415-008-3004-y, indexed in Pubmed: 18685921.

26. Magraner MJ, Coret F, Casanova B. The effect of intravenous immunoglobulin on neuromyelitis optica. Neurologia. 2013; 28(2): 65-72, doi: 10.1016/j.nrl.2012.03.014, indexed in Pubmed: 22841880.

27. Viswanathan S, Wong AHY, Quek AML, et al. Intravenous immunoglobulin may reduce relapse frequency in neuromyelitis optica. J Neuroimmunol. 2015; 282: 92-96, doi: 10.1016/j.jneuroim.2015.03.021, indexed in Pubmed: 25903734.

28. Cabre P, Mejdoubi M, Jeannin S, et al. Francophone Society of Multiple Sclerosis and OFSEP investigators. Treatment of neuromyelitis optica with rituximab: a 2-year prospective multicenter study. J Neurol. 2018; 265(4): 917-925, doi: 10.1007/s00415-018-8771-5, indexed in Pubmed: 29455361.

29. Alldredge B, Jordan A, Imitola J, et al. Safety and Efficacy of Rituximab: Experience of a Single Multiple Sclerosis Center. Clin Neuropharmacol. 2018; 41(2): 56-59, doi: 10.1097/WNF.0000000000000268, indexed in Pubmed: 29389745.

30. Gao F, Chai B, Gu C, et al. Effectiveness of rituximab in neuromyelitis optica: a meta-analysis. BMC Neurol. 2019; 19(1): 36, doi: 10.1186/ s12883-019-1261-2, indexed in Pubmed: 30841862.

31. Brachet G, Bourquard T, Gallay N, et al. Eculizumab epitope on complement C5: Progress towards a better understanding of the mechanism of action. Mol Immunol. 2016; 77: 126-131, doi: 10.1016/j. molimm.2016.07.016, indexed in Pubmed: 27497837.

32. Soltys J, Liu Y, Ritchie A, et al. Membrane assembly of aquaporin-4 autoantibodies regulates classical complement activation in neuromyelitis optica. J Clin Invest. 2019; 129(5): 2000-2013, doi: 10.1172/ JCl122942, indexed in Pubmed: 30958797.

33. Yao X, Verkman AS. Complement regulator CD59 prevents peripheral organ injury in rats made seropositive for neuromyelitis optica immunoglobulin G. Acta Neuropathol Commun. 2017; 5(1): 57, doi: 10.1186/s40478-017-0462-4, indexed in Pubmed: 28750658.

34. Pittock SJ, Berthele A, Fujihara K, et al. Eculizumab in Aquaporin-4-Positive Neuromyelitis Optica Spectrum Disorder. N Engl J Med. 2019; 381(7): 614-625, doi: 10.1056/NEJMoa1900866, indexed in Pubmed: 31050279. 
35. Benamu E, Montoya JG. Infections associated with the use of eculizumab: recommendations for prevention and prophylaxis. Curr Opin Infect Dis. 2016; 29(4): 319-329, doi: 10.1097/ QC0.0000000000000279, indexed in Pubmed: 27257797.

36. Horellou P, Wang M, Keo V, et al. Increased interleukin-6 correlates with myelin oligodendrocyte glycoprotein antibodies in pediatric monophasic demyelinating diseases and multiple sclerosis. J Neuroimmunol. 2015; 289: 1-7, doi: 10.1016/j.jneuroim.2015.10.002, indexed in Pubmed: 26616865.

37. Kieseier BC, Stüve O, Dehmel T, et al. Disease amelioration with tocilizumab in a treatment-resistant patient with neuromyelitis optica: implication for cellular immune responses. JAMA Neurol. 2013; 70(3): 390-393, doi: 10.1001/jamaneurol.2013.668, indexed in Pubmed: 23599943.

38. Araki M, Matsuoka T, Miyamoto K, et al. Efficacy of the anti-IL-6 receptor antibody tocilizumab in neuromyelitis optica: a pilot study. Neurology. 2014; 82(15): 1302-1306, doi: 10.1212/ WNL.0000000000000317, indexed in Pubmed: 24634453.

39. Ringelstein M, Ayzenberg I, Harmel J, et al. Long-term Therapy With Interleukin 6 Receptor Blockade in Highly Active Neuromyelitis Optica Spectrum Disorder. JAMA Neurol. 2015; 72(7): 756-763, doi: 10.1001/jamaneurol.2015.0533, indexed in Pubmed: 25985228.

40. Zhang C, Zhang M, Qiu W, et al. Tocilizumab versus Azathioprine in highly relapsing neuromyelitis optica spectrum disorders (TANGO): a head-to-head comparative study. Multiple Sclerosis J. 2019; 25(S2): 44.

41. Kaplon H, Reichert JM. Antibodies to watch in 2018. MAbs. 2018; 10(2): 183-203, doi: 10.1080/19420862.2018.1415671, indexed in Pubmed: 29300693.

42. Ayzenberg I, Kleiter I, Schröder A, et al. Interleukin 6 receptor blockade in patients with neuromyelitis optica nonresponsive to anti-CD20 therapy. JAMA Neurol. 2013; 70(3): 394-397, doi: 10.1001/jamaneurol.2013.1246, indexed in Pubmed: 23358868.

43. Uchida T, Mori M, Uzawa A, et al. Increased cerebrospinal fluid metalloproteinase-2 and interleukin- 6 are associated with albumin quotient in neuromyelitis optica: Their possible role on blood-brain barrier disruption. Mult Scler. 2017; 23(8): 1072-1084, doi: 10.1177/1352458516672015, indexed in Pubmed: 27682231.

44. Kimura A, Naka T, Kishimoto T. IL-6-dependent and -independent pathways in the development of interleukin 17-producing T helper cells. Proc Natl Acad Sci U S A. 2007; 104(29): 12099-12104, doi: 10.1073/pnas.0705268104, indexed in Pubmed: 17623780.

45. Igawa $\mathrm{T}$, Ishii $\mathrm{S}$, Tachibana $\mathrm{T}$, et al. Antibody recycling by engineered pH-dependent antigen binding improves the duration of antigen neutralization. Nat Biotechnol. 2010; 28(11): 1203-1207, doi: 10.1038/ nbt.1691, indexed in Pubmed: 20953198.

46. de Se, Kleiter I, Fujihara K, et al. et al.. A double-blind placebo-controlled study of satralizumab (SA237), a recycling anti-IL-6 receptor monoclonal antibody, as add-on therapy for neuromyelitis optica spectrum disorder (NMOSD). MS Journal 2018, 24: issue 2 suppl. : abstract.

47. Bennett, B. Greenberg, A. Traboulsee, Efficacy of satralizumab as monotherapy in pre-specified subgroups of SAkuraStar, a double-blind placebo-controlled Phase 3 clinical study in patients with neuromyelitis optica spectrum disorder (NMOSD)J.L. Multiple Sclerosis J. 2019; 25(S2): 44.

48. Chen D, Gallagher S, Monson NL, et al. Inebilizumab, a B Cell-Depleting Anti-CD19 Antibody for the Treatment of Autoimmune Neurological Diseases: Insights from Preclinical Studies. J Clin Med. 2016; 5(12), doi: 10.3390/jcm5120107, indexed in Pubmed: 27886126 .
49. Cree BAC, Bennett JL, Kim HoJ, et al. N-MOmentum study investigators. Inebilizumab for the treatment of neuromyelitis optica spectrum disorder (N-MOmentum): a double-blind, randomised placebo-controlled phase 2/3 trial. Lancet. 2019; 394(10206): 1352-1363, doi: 10.1016/S0140-6736(19)31817-3, indexed in Pubmed: 31495497.

50. Jarius S, Paul F, Franciotta D, et al. Cerebrospinal fluid findings in aquaporin-4 antibody positive neuromyelitis optica: results from 211 lumbar punctures. J Neurol Sci. 2011; 306(1-2): 82-90, doi: 10.1016/j.jns.2011.03.038, indexed in Pubmed: 21550068.

51. Michael BD, Elsone L, Griffiths MJ, et al. Post-acute serum eosinophil and neutrophil-associated cytokine/chemokine profile can distinguish between patients with neuromyelitis optica and multiple sclerosis; and identifies potential pathophysiological mechanisms - a pilot study. Cytokine. 2013; 64(1): 90-96, doi: 10.1016/j.cyto.2013.07.019, indexed in Pubmed: 23941778.

52. Tradtrantip L, Ratelade J, Zhang $\mathrm{H}$, et al. Enzymatic deglycosylation converts pathogenic neuromyelitis optica anti-aquaporin-4 immunoglobulin G into therapeutic antibody. Ann Neurol. 2013; 73(1): 77-85, doi: 10.1002/ana.23741, indexed in Pubmed: 23055279.

53. Tradtrantip L, Asavapanumas N, Verkman AS. Therapeutic cleavage of anti-aquaporin-4 autoantibody in neuromyelitis optica by an IgG-selective proteinase. Mol Pharmacol. 2013; 83(6): 1268-1275, doi: 10.1124/mol.113.086470, indexed in Pubmed: 23571414.

54. Verkman AS, Phuan PW, Asavapanumas N, et al. Biology of AQP4 and anti-AQP4 antibody: therapeutic implications for NMO. Brain Pathol. 2013; 23(6): 684-695, doi: 10.1111/bpa.12085, indexed in Pubmed: 24118484.

55. Verkman AS, Smith AJ, Phuan PW, et al. The aquaporin-4 water channel as a potential drug target in neurological disorders. Expert Opin Ther Targets. 2017; 21(12): 1161-1170, doi: 10.1080/14728222.2017.1398236, indexed in Pubmed: 29072508.

56. Saadoun S, Waters P, MacDonald C, et al. Neutrophil protease inhibition reduces neuromyelitis optica-immunoglobulin G-induced damage in mouse brain. Ann Neurol. 2012; 71(3): 323-333, doi: 10.1002/ ana.22686, indexed in Pubmed: 22374891.

57. Herges K, de Jong BA, Kolkowitz I, et al. Protective effect of an elastase inhibitor in a neuromyelitis optica-like disease driven by a peptide of myelin oligodendroglial glycoprotein. Mult Scler. 2012; 18(4): 398-408, doi: 10.1177/1352458512440060, indexed in Pubmed: 22343184.

58. Domon H, Nagai K, Maekawa T, et al. Neutrophil Elastase Subverts the Immune Response by Cleaving Toll-Like Receptors and Cytokines in Pneumococcal Pneumonia. Front Immunol. 2018; 9: 732, doi: 10.3389/fimmu.2018.00732, indexed in Pubmed: 29922273.

59. Muroishi T, Sakai K, Yanase D, et al. Serum anti-aquaporin-4 antibody-positive neuromyelitis optica spectrum disorder presenting as acute eosinophilic encephalomyelitis. J Clin Neurosci. 2018; 48: 93-94, doi: 10.1016/j.jocn.2017.10.074, indexed in Pubmed: 29137920.

60. Zhang H, Verkman AS. Eosinophil pathogenicity mechanisms and therapeutics in neuromyelitis optica. J Clin Invest. 2013; 123(5): 2306 2316, doi: 10.1172/JCl67554, indexed in Pubmed: 23563310.

61. Katz Sand I, Fabian MT, Telford R, et al. Open-label, add-on trial of cetirizine for neuromyelitis optica. Neurol Neuroimmunol Neuroinflamm. 2018; 5(2): e441, doi: 10.1212/NXI.0000000000000441, indexed in Pubmed: 30426035.

62. Zhang C, Tian DC, Yang CS, et al. Safety and Efficacy of Bortezomib in Patients With Highly Relapsing Neuromyelitis Optica Spectrum Disorder. JAMA Neurol. 2017; 74(8): 1010-1012, doi: 10.1001/jamaneurol.2017.1336, indexed in Pubmed: 28692708. 